\title{
The Importance of High-Resolution Scanning Transmission Electron Microscopy For Fine-Scale Dislocation Analysis
}

\author{
P. J. Phillips*, L. Kovarik*, R. R. Unocic**, D. Wei***, D. Mourer***, M. J. Mills* \\ *Ohio State University, 2041 N College Rd, Columbus, OH 43210 \\ **Oak Ridge National Lab, 1 Bethel Valley Rd. Oak Ridge, TN 37831-6064 \\ ***GE Aviation, Lynn, MA 01910
}

Ni-based superalloys are currently used in the hot section of turbine engines due to their retained high-temperature strength under extreme environments. Extensive, detailed work has been done on the deformation mechanisms of these materials under creep conditions [1]; however, in the area of low cycle fatigue (LCF) the same detailed characterization is somewhat lacking and the deformation is not well understood. High temperature cyclic softening [2] is observed during LCF of the polycrystalline disc superalloy R104. In general, it is accepted that planar anti-phase boundary (APB) shearing of the strengthening $\gamma^{\prime}$-precipitates, followed by their dissolution, is the principal cause of cyclic softening [3]. Given the precipitate morphology and bimodal microstructure of R104, this mechanism is unlikely to be responsible for cyclic softening as the $\gamma^{\prime}$ will not be sheared away after a nominal number of cycles; therefore there must exist another principal cause.

Following $\sim 1300$ fatigue cycles at $704{ }^{\circ} \mathrm{C}$, initial dislocation analysis was performed on an FEI Tecnai F20 S/TEM operated at $200 \mathrm{kV}$. $\{111\}$ cross-slip and cube-slip were found to be the most prevalent deformation mechanisms (Fig. 1). Note the fine-scale, triangular features of contrast that appear both on cube planes, at intersections of $\{111\}$ dislocations, and as semi-isolated pairs [4]. Given the size scale of these features, traditional STEM methods were unable to provide sufficient resolution, and thus their true nature could not be determined. High-resolution microscopy was then performed on an FEI $\operatorname{Titan}^{3}$ 80-300 probe-corrected monochromated S/TEM operated at $300 \mathrm{kV}$. The microscope was operated in both high- and low-angle annular dark field (HAADF and LAADF) imaging modes, with LAADF conditions optimized in order to retain directly-interpretable images while enhancing the strain-contrast from defect structures. As such, optimum microscope conditions in terms of camera length (collection angle) will be discussed.

Only with the aid of atomic resolution microscopy were the following two distinct types of dissociations found. The first: stacking fault tetrahedra and the second: Lomer-Cottrell locks on cube-plane dislocations. Figure 2a details examples of the tetrahedra, which are often found in pairs, likely indicative of the paired dislocations that preceded them cooperatively shearing $\gamma^{\prime}$. A potential mechanism of tetrahedra formation is discussed in [5], in which ultimately a planar Frank Loop "unzips" to form a three-dimensional tetrahedron. While this mechanism is likely, work is in progress to definitively determine the process of formation. Figure $2 b$ is one example of the socalled Lomer-Cottrell lock. The dislocation shown is the upper-right feature of the inset image, which contains two pairs of dislocations lying in the cube plane. All four dislocations of the inset image contain the two extra $\{111\}$ half-planes definitive of a Lomer-Cottrell configuration.

While the role of these dissociations in cube-slip and cyclic softening is not fully understood, their frequent occurrence suggests that these processes are certainly relevant at this stage of deformation. It is important to note that without employing high-resolution microscopy, these features could easily have been incorrectly identified, or missed entirely, and thus the importance of atomic resolution STEM in dislocation analysis cannot be overlooked. 
[1] L. Kovarik, et al., Prog. Mater. Sci. 54 (2009) 839-873.

[2] D. P. Mourer, GEAE, 2006. Private Communication.

[3] M. Sundararaman, et al., Scripta Metall. 23 (1989) 1795-1800.

[4] P.J. Phillips, et al., Scripta Mater. (2010), doi:10.1016/j.scriptamat.2010.01.044.

[5] J. Hirth and J. Lothe, Theory of Dislocations, second ed., Wiley 1982.

[6] Support was provided by the University Strategic Alliance (USA) Program, GE Aviation

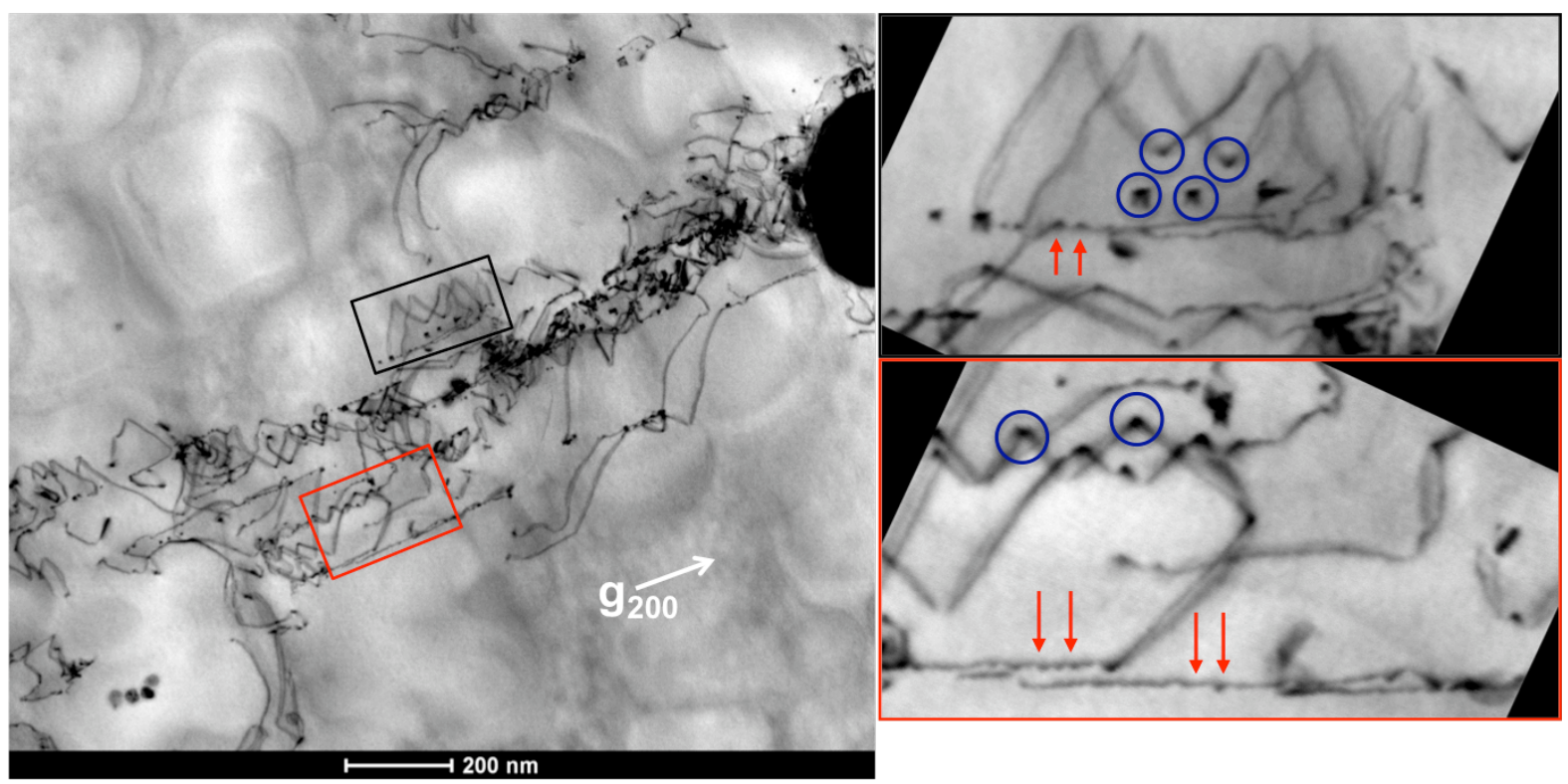

Fig. 1. BF STEM images of dislocation substructure: ample dislocations either on cube planes or cross-slipping on $\{111\}$ planes. Note the features of triangular contrast in the right images.

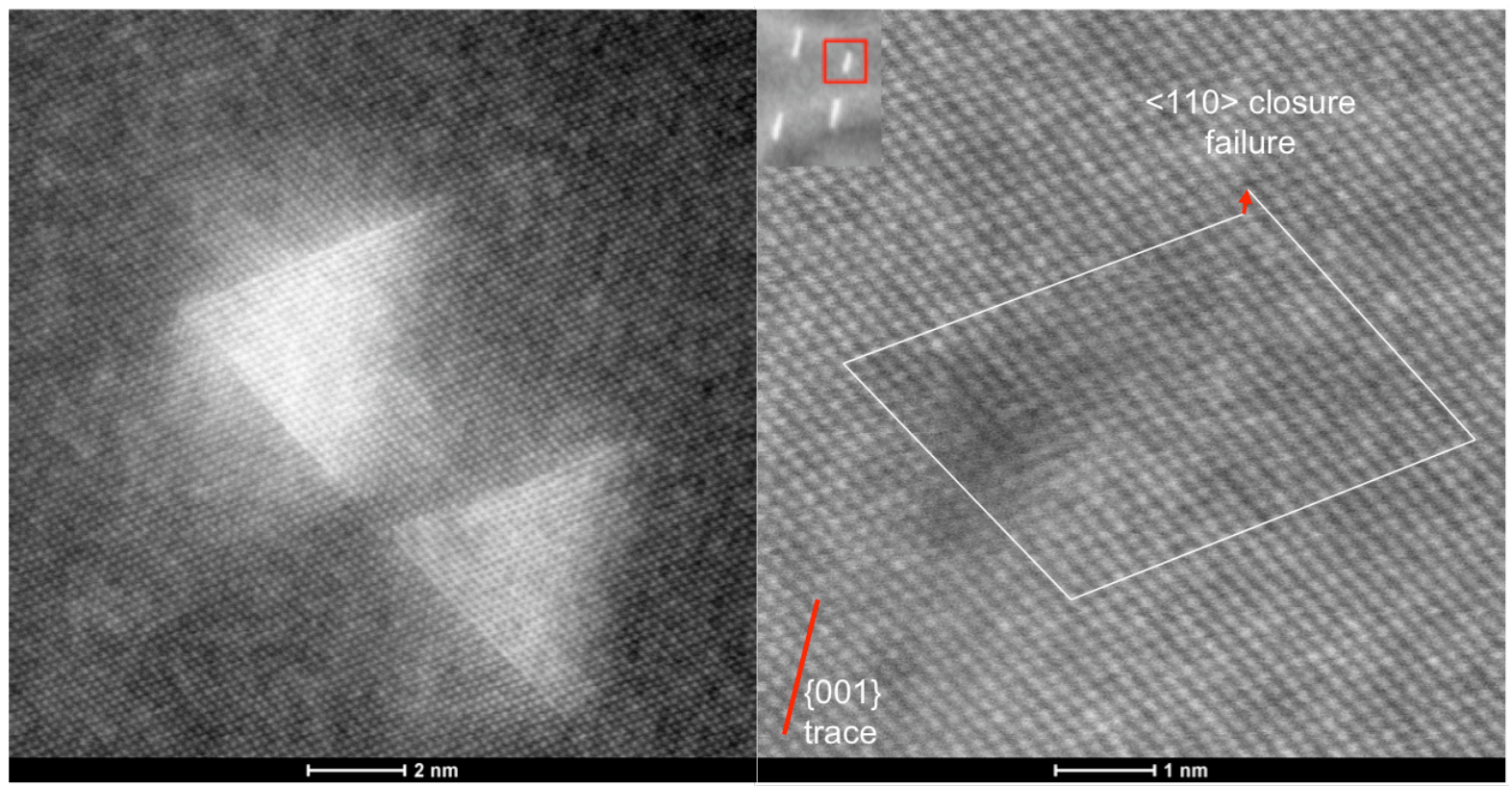

Fig. 2. left) LAADF image of two stacking fault tetrahedra; right) HAADF image of cube-plane dislocation (upper right of inset); Burgers circuit analysis reveals extra half-planes in each $\{111\}$ plane. Note the indicated cube plane trace is valid for both images. 